\title{
Eficiência do diagnóstico coproscópico de Schistosoma mansoni em fezes prensadas
}

\author{
The efficiency of the examination of compressed fecal samples \\ for Schistosoma mansoni eggs \\ Horacio Manuel Santana Teles', Cláudio Santos Ferreira², Maria Esther de Carvalho, \\ Fabiana Zacharias ${ }^{1}$ e Luiz Augusto Magalhães ${ }^{3}$
}

\begin{abstract}
Resumo A eficácia do controle da esquistossomose depende em grande parte da sensibilidade da coproscopia. Passamos a utilizar, além da técnica de Kato-Katz, a de prensagem de fezes entre lâmina e lamínula de polipropileno, segundo Ferreira. De 1.282 amostras fecais colhidas entre 1998 e 2000 no Bairro da Palha, Município de Bananal, São Paulo, Brasil, 105 (8,2\%) resultaram positivas. A primeira técnica revelou 90 (7\%) e a segunda, 88 (6,9\%) amostras positivas. Os resultados concordaram, segundo a estatística kappa. Valores baixos de percentagens de positivos e de opg (ovos por grama) de fezes, em Bananal, demandaram aumento do volume de material a examinar. Além de permitir a observação da viabilidade dos ovos de Schistosoma mansoni, a segunda técnica dispensa o uso de glicerina, de placa medidora e de tamisação; esta última, responsável por ulterior perda de precisão nas determinações de opg.
\end{abstract}

Palavras-chaves:Schistosoma mansoni. Diagnóstico coproscópico. Sensibilidade. Esquistossomose. Morbidade.

Abstract The control of schistosomiasis depends mostly upon the sensitivity of stool examinations. We used both the Kato-Katz technique and the compression of samples between the slide and a polypropylene cover glass, according to Ferreira. Out of 1,282 samples collected between 1998 and 2000 in the Palha District, Municipality of Bananal, São Paulo State, Brazil, 105 (8.2\%) were positive. The first and second methods revealed 90 (7\%) and 88 (6.9\%) positive cases, respectively. According to the kappa statistic, both methods were in agreement. In Bananal, the proportion of positive cases and egg per gram (epg) values are low, which calls for the examination of larger than usual volumes of feces. The viability of Schistosoma mansoni eggs can also be checked when using the second method, which dispenses with glycerin solution, measuring plates and sieves. The latter cause a further loss of precision in epg determinations.

Key-words: Schistosoma mansoni. Fecal examination. Sensitivity. Schistosomiasis. Morbidity.

O diagnóstico morfológico dos ovos de Schistosoma mansoni em amostras de fezes prensadas é uma das linhas de base da execução dos programas de controle da transmissão da esquistossomose mansônica, endemia ainda bastante freqüente em várias regiões do território brasileiro (SUCAM ${ }^{20}{ }^{21}$ ). Afora a improbabilidade de falsos positivos, simplicidade e baixo custo constituem as principais vantagens do uso da coproscopia em inquéritos destinados à busca de portadores do parasita para ulterior tratamento.

A técnica usualmente adotada nos programas de controle da endemia é a de Kato \& Miura ${ }^{22}$ apud Komiya \& Kobayashi ${ }^{14}$ que, modificada por Katz et $a^{l^{13}}$, passou a incluir o uso de um tamís para a remoção de resíduos macroscópicos e de placa medidora para controlar o volume das amostras fecais a examinar. Por permitir a obtenção de dados quantitativos em termos de ovos por grama de fezes (opg), o assim chamado método de Kato-Katz passou a ser recomendado pela Organização Mundial de Saúde ${ }^{22}$ e por vários autores, dentre eles Mott ${ }^{18}$, para emprego em programas de controle da esquistossomose mansônica. Anteriormente, Martin \& Beaver ${ }^{16}$, Chaia et $a^{4}$, Molina ${ }^{17}$, Coura \& Conceição ${ }^{7} \mathrm{e}$ Dantas \& Ferreira $^{8}$ haviam discutido vantagens e desvantagens de outras técnicas de diagnostico coproscópico de S. mansoni.

\footnotetext{
1. Superintendência de Controle de Endemias, SUCEN, São Paulo, SP. 2. Laboratório de Investigação Médica do Hospital das Clínicas da Faculdade de Medicina da Universidade de São Paulo, São Paulo, SP. 3. Instituto de Biologia do Departamento de Parasitologia da Universidade de Campinas, Campinas, SP.

Endereço para correspondência: Dr. Horacio Manuel Santana Teles. Rua Paula Souza 166, 01027-000 São Paulo, SP, Brasil.

Tel: 5511 227-0622, Fax: $5511229-8292$.

e-mail:horacio@sucen.sp.gov.br

Recebido para publicação em 13/8/2001

Aceito em 6/6/2003
} 
Em 1978, o Centro de Informações da Saúde (CIS) ${ }^{6}$ introduziu o método de Kato-Katz como procedimento de rotina no diagnóstico laboratorial da esquistossomose, além da técnica de sedimentação por gravidade descrita por Lutz ${ }^{15}$ e depois por Hoffman, Pons \& Janer ${ }^{11}$, visando a reduzir a proporção da falsos negativos. A par dessa recomendação, o relatório da Superintendência de Controle de Endemias (SUCEN ${ }^{21}$ ), sobre a situação epidemiológica da esquistossomose em território paulista, indica que o exame de amostras pela técnica de fezes prensadas já era utilizado em inquéritos coproscópicos de migrantes originários de áreas endêmicas situadas fora do Estado de São Paulo.

É necessário, indiscutivelmente, aprimorar as técnicas em uso, tendo-se em conta, além da redução da prevalência, a das cargas parasitárias. É importante salientar que a fácil identificação dos ovos de $S$. mansoni torna improvável a ocorrência de resultados falsospositivos. Por outro lado, a relativamente baixa sensibilidade das técnicas de demonstração direta de formas evolutivas de parasitas pode ser atenuada por meio de refinamentos que aumentem as probabilidades de seu encontro em material onde sejam escassas. Para isto é freqüentemente necessário usar mais de uma técnica coproscópica. Dentre os fatores a ponderar, ao ser feita a escolha de procedimentos para uso rotineiro está a relação custo-benefício; é oportuno salientar que algumas técnicas tornaram-se desnecessariamente onerosas. O presente estudo é conseqüência de investigação deste problema; inclui a análise de resultados do emprego, dentro dos programas de controle da esquistossomose em São Paulo, de duas técnicas: a adotada rotineiramente e uma alternativa, ambas consistindo no exame de fezes prensadas.

\section{MATERIAL E MÉTODOS}

As amostras fecais foram colhidas por ocasião de três inquéritos coproscópicos da população residente no Bairro da Palha, como previsto no Plano de Intensificação do Controle da Esquistossomose Mansônica no Município de Bananal, São Paulo, Brasil, no período de 1998 a 2000. Uma equipe local da SUCEN executou os trabalhos de campo: distribuiu recipientes e os recolheu com as amostras de fezes. Chegaram ao laboratório 1282 amostras, processadas segundo as técnicas de KatoKatz e de Ferreira apud Araújo². No primeiro caso, empregou-se o kit $\mathrm{AK}^{\circledR}$. Preparadas duas lâminas para a leitura, obteve-se o número de ovos por grama de fezes (opg) por meio do uso da tabela Vermi-Fec ${ }^{\circledR}$ que acompanha o kit. Pressupondo-se que a quantidade de fezes contidas no medidor corresponda a $41,7 \mathrm{mg}^{722}$, o valor mínimo de opg será 12. Amato Neto \& Corrêa ${ }^{1}$ descrevem pormenorizadamente o método de Kato-Katz.

A técnica de Ferreira apud Araújo $^{2}$, dispensa a tamisação, o uso de glicerina e placas medidoras. Transferem-se para a lâmina pequenas porções de fezes, sendo os resíduos macroscópicos afastados por meio de catação. A quantidade a ser transferida é avaliada por observação visual, de modo a permitir a obtenção de esfregaço fecal de tamanho conveniente, após compressão (em torno de 18mm de diâmetro). Após a transferência da amostra de fezes para a lâmina, coloca-se sobre esta uma lamínula de polipropileno incolor, de aproximadamente 24 por $24 \mathrm{~mm}$, fazendose então a compressão de modo semelhante ao usado na técnica de Kato-Katz. O exame pode ser feito imediatamente. Os volumes fecais neste caso são menores do que os usados nas preparações segundo Kato-Katz, tendo sido examinadas três lâminas de cada amostra. Para o cálculo do volume das fezes medemse dois diâmetros do esfregaço perpendiculares entre si. A média aritmética desses diâmetros é usada para, com o auxílio de uma tabela, achar o fator a ser usado para calcular opg. A tabela teve como base o cálculo de regressão em que a variável independente foi o volume de fezes, medido em placa medidora (entre 13,6 e $55,3 \mathrm{~mm}^{3}$ ) e a variável dependente o diâmetro do esfregaço prensado (em $\mathrm{mm}$ ). Para o cálculo do volume a partir do diâmetro médio do esfregaço, usa-se a equação: $Y=A+B X$, onde $Y=\ln$ y (volume em $\mathrm{mm}^{3}$ ), $X=\ln x$ (diâmetro em $\mathrm{mm}$ ), $A=\ln$ a e $B=b$, sendo $a=$ $4,263 \times 10^{-3}$ (coeficiente linear) e $b=2,980$ (coeficiente angular). A tabela pode ser facilmente obtida com o uso de planilha eletrônica.

\section{RESULTADOS}

Como se observa nas Tabelas 1 e 2, pela técnica de Katz et $a^{\prime / 3}$ os exames revelaram 31 amostras positivas em 1998, 36 em 1999 e 23 em 2000, o que respectivamente corresponde a $8,1 \%, 8,0 \%$ e $5,2 \%$ do total de amostras colhidas a cada inquérito, enquanto pela técnica de Ferreira apud Araújo ${ }^{1}$, foram positivas 28, 40 e 20 amostras, isto é, 7,3\%, 8,8\% e 4,5\%. As amostras positivas para ovos de $S$. mansoni em ambas as ténicas foram 34 dentre 384 amostras de fezes examinadas em 1998, 46 dentre 455 em 1999 e 25 dentre 443 em 2000 ou, respectivamente, $8,8 \%, 10,1 \%$ e $5,6 \%$ de positividade. A concordância anual em números absolutos foi de 25, 30 e 18 casos, como se deduz da Tabela 1. Assim, os percentuais de concordância bruta foram de $97,7 \%$ em 1998 , 96,5\% em 1999 e 98,4\% em 2000. Durante os 3 anos da avaliação, a técnica de diagnóstico coproscópico desenvolvida por Katz et a ${ }^{13}$ possibilitou o diagnóstico de 90 portadores de $S$. mansoni e a de Ferreira apud Araújo ${ }^{2}$ revelou 88 casos. Por ambas as técnicas foram detectados 105 casos de esquistossomose entre a população examinada nos 3 inquéritos. Aplicada a estatística kappa, para a verificação da concordância sem o efeito do acaso, obtêm-se respectivamente os 
Tabela 1 - Positividade para ovos de S. mansoni nas amostras de fezes procedentes do Bairro da Palha, Município de Bananal, Estado de São Paulo, Brasil de 1998 a 2000.

\begin{tabular}{|c|c|c|c|c|c|c|c|c|c|}
\hline \multirow{2}{*}{$\begin{array}{l}\text { Ano } \\
\text { Método }\end{array}$} & \multicolumn{3}{|c|}{1998} & \multicolumn{3}{|c|}{1999} & \multicolumn{3}{|c|}{2000} \\
\hline & $\mathrm{K}(+) \mathrm{F}(+)$ & $\mathrm{K}(+) \mathrm{F}(-)$ & $\mathrm{K}(-) \mathrm{F}(+)$ & $\mathrm{K}(+) \mathrm{F}(+)$ & $\mathrm{K}(+) \mathrm{F}(-)$ & $\mathrm{K}(-) \mathrm{F}(+)$ & $\mathrm{K}(+) \mathrm{F}(+)$ & $\mathrm{K}(+) \mathrm{F}(-)$ & $\mathrm{K}(-) \mathrm{F}(+)$ \\
\hline$\overline{(+)}$ & 25 & 6 & 3 & 30 & 6 & 10 & 23 & 5 & 2 \\
\hline$(-)$ & & 350 & & & 409 & & & 418 & \\
\hline Total & & 384 & & & 455 & & & 443 & \\
\hline
\end{tabular}

K=técnica de Kato \& Miura ${ }^{11}$ modificada por Katz et $a^{1 / 3}$

F=técnica de Ferreira apud Araújo²

(+)amostra positiva para ovos de S. mansoni

(-)amostra negativa para ovos de S. mansoni

Tabela 2 - Total de exames realizados e de amostras positivas e negativas para ovos de S. mansoni no Bairro da Palha, Município de Bananal, Estado de São Paulo, Brasil de 1998 a 2000.

\begin{tabular}{|c|c|c|c|c|c|c|}
\hline \multirow{2}{*}{$\begin{array}{l}\text { Ano } \\
\text { Método }\end{array}$} & \multicolumn{2}{|c|}{$\begin{array}{c}1998 \\
\mathrm{n}=384\end{array}$} & \multicolumn{2}{|c|}{$\begin{array}{c}1999 \\
\mathrm{n}=455\end{array}$} & \multicolumn{2}{|c|}{$\begin{array}{c}2000 \\
n=443\end{array}$} \\
\hline & K (\%) & $F(\%)$ & $\mathrm{K}(\%)$ & $F(\%)$ & $\mathrm{K}(\%)$ & $F(\%)$ \\
\hline$(+)$ & $31(8,1)$ & $28(7,3)$ & $36(7,9)$ & $40(8,8)$ & $23(5,2)$ & $20(4,5)$ \\
\hline$(-)$ & $353(91,9)$ & $356(92,7)$ & $419(92,1)$ & $415(91,2)$ & $420(94,8)$ & $423(95,5)$ \\
\hline
\end{tabular}

$\mathrm{K}=$ técnica de Kato \& Miura ${ }^{11}$ modificada por Katz et al.

F=técnica de Ferreira apud Araújo².

valores $0,835,0,770$ e 0,829 , correspondentes aos graus de concordância quase perfeita no primeiro e no terceiro inquéritos e substancial no segundo.

Quanto às cargas parasitárias estimadas em termos de opg, as médias obtidas por meio da técnica usual dos programas de controle da esquistossomose e da segunda foram, respectivamente: 105,3 e 220,3 em 1998, 72,5 e 72,5 em 1999 e 78,9 e 107 em 2000.

A Tabela 3 apresenta a distribuição das cargas parasitárias em intervalos que caracterizam a possibilidade do desenvolvimento das formas leves, moderadas ou intensas da doença, tendo em vista a probabilidade da ocorrência da hepatomegalia. Os parâmetros para as estimativas da morbidade indicados pela Organização Mundial de Saúde $\left(\mathrm{OMS}^{22}\right)$ foram modificados nos limites inferiores de opg, dado o aumento do volume de fezes examinado por nós, correspondente a duas lâminas por amostra de fezes. $\mathrm{Na}$ Tabela 3 as cargas parasitárias estão distribuídas por classes, tendo-se em vista sua relação com a probabilidade de ocorrência de formas clínicas graves.

\begin{tabular}{|c|c|c|c|c|c|c|}
\hline \multirow{2}{*}{$\begin{array}{l}\text { Ano } \\
\text { Método }\end{array}$} & \multicolumn{2}{|c|}{1998} & \multicolumn{2}{|c|}{1999} & \multicolumn{2}{|c|}{2000} \\
\hline & $\mathrm{K}(\%)$ & $F(\%)$ & K (\%) & $F(\%)$ & $\mathrm{K}(\%)$ & $F(\%)$ \\
\hline Até 96 opg* & $22(71,0)$ & $13(46,4)$ & $30(83,3)$ & $32(80,0)$ & $21(91,3)$ & $8(40,0)$ \\
\hline $96<0 \mathrm{pg}<792^{*}$ & $8(25,8)$ & $13(46,4)$ & $6(17,7)$ & $8(20,0)$ & $2(8,7)$ & $10(50,0)$ \\
\hline$>792$ ogp* & $1(3,2)$ & $2(7,1)$ & $0(0,0)$ & $0(0,0)$ & $0(0,0)$ & $2(10,0)$ \\
\hline Total & $31(100,0)$ & $28(100,0)$ & $36(100,0)$ & $40(100,0)$ & $23(100,0)$ & $20(100,0)$ \\
\hline
\end{tabular}

(") Modificado de $\mathrm{WHO}^{22}$

$\mathrm{K}=$ técnica de Kato \& Miura ${ }^{11}$ modificada por Katz et $a^{{ }^{13}}$

$\mathrm{F}=$ técnica de Ferreira apud Araújo

\section{DISCUSSÃO}

A pesquisa de formas evolutivas de parasitas em amostras de fezes tem pontos positivos inegáveis, dentre elas a improbabilidade de falsos resultados positivos, baixo custo e possibilidade de ser executada em laboratórios com escassos recursos. Não compete necessariamente com os métodos indiretos, de introdução recente, indicados para assinalar a presença de parasitas em seus hospedeiros, S. mansoni inclusive.
Chieffi \& Kanamura ${ }^{5}$, Barreto et al ${ }^{3}$, Rabelo ${ }^{19}$, Gryssels $^{10}$ e Engels et $a^{\beta}$ discutiram causas da baixa eficiência e sensibilidade do diagnóstico coproscópico de $S$. mansoni relacionados com a biologia do parasita, destacando a ocorrência de infecções por pequeno número de vermes, eventualmente unissexuadas, irregularidade das posturas, distribuição heterogênea dos ovos no bolo fecal e desenvolvimento de imunidade por 
parte do hospedeiro. Tais fatores, bem conhecidos, podem ser compensados em parte, segundo a $\mathrm{OMS}^{22}$, com a utilização de maiores volumes do material a examinar ou colheita de mais de uma amostra por pessoa, dentro de pequenos intervalos de tempo. A colheita de várias amostras por pessoa pode aumentar consideravelmente os custos operacionais. Entretanto, tendo como base processos simples, é possível refinar as técnicas de coproscopia, treinar mais adequadamente o pessoal, demonstrando as bases teóricas das técnicas adotadas e o uso correto do equipamento, incluindo-se aí o microscópio. Todas as operações incluídas nos exames coproscópicos devem ser rigorosamente padronizadas, regra a ser aplicada a quaisquer outras técnicas de laboratório. O uso da coproscopia não exclui a adoção criteriosa de exames clínicos e laboratoriais, indicados para subsidiar os estudos epidemiológicos. No caso em questão, mesmo sendo admitidas as limitações da coproscopia, sua aplicação permitiu a identificação de problemas práticos e o delineamento de medidas profiláticas. Os dois processos empregados mostraram resultados equivalentes. A técnica de Ferreira apud Araújo², por não acrescentar reagentes à massa fecal, permite observar os miracídios no interior dos ovos. Para facilitar a observação dos parasitas, recomendase usar, em cada lâmina, quantidades de material menores do que as usadas no processo de Kato-Katz, devendo ser portanto, examinado número correspondentemente maior de lâminas.

A comparação dos resultados de contagens em termos de opg revelou diferenças significativas quanto à avaliação em termos de gravidade (Tabela 3). O fato é importante porque dificulta avaliações, nos programas de controle, de riscos do desenvolvimento de formas graves da esquistossomose mansônica. Tais diferenças podem ser atribuídas ao fato de terem sido pequenas, em média, as quantidades de ovos eliminados. Devese ter em mente o fato de os resultados de exames de laboratório fornecerem subídios para a interpretação de problemas clínicos ou epidemiológicos; o diagnóstico de cada situação resulta de dados provenientes de muitas outras fontes. Os programas de controle, portanto, são dimensionados à base de informações válidas para populações, não necessáriamente para casos individuais, aos quais podem aplicar-se técnicas semiológicas especificamente indicadas como, entre muitas outras, a ultrassonografia $\left(\mathrm{OMS}^{23}\right)$.

Nas condições estabelecidas para a presente pesquisa, a comparação dos resultados obtidos com a aplicação das técnicas desenvolvidas por Katz et a/ ${ }^{13} \mathrm{e}$ Ferreira apud Araújo², conclui-se que a segunda técnica, embora ainda não utilizada em grande escala tem as vantagens de ser mais simples e econômica, além de permitir observar a viabilidade dos ovos de S. mansoni. Quanto à tamisação das fezes, esta representa uma ponderável causa de erro em contagens, tendendo a superestimar os valores de opg, por motivos óbvios, embora possa ter influência positiva em exames qualitativos. Essa etapa pode ser suprimida quando realizados exames quantitativos.

\section{AGRADECIMENTOS}

Os autores agradecem as inestimáveis colaborações do Prof. Dr. Vicente Amato Neto do Laboratório de Investigação Médica, Parasitologia do Hospital das Clínicas da Medicina Tropical da FM/USP, da Profá . Hilda Maria Solís Acosta da Universidad Mayor de San Marcos, Lima, Peru, dos técnicos Maria Diniz Barbosa e Valdecir Rodrigues de Almeida do Serviço Regional de Taubaté da SUCEN e das estagiárias da FUNDAP na SUCEN, Andréa Moreira da Cunha e Valquíria Rosa de Lima.

\section{REFERÊNCIAS BIBLIOGRÁFICAS}

1. Amato Neto V, Corrêa LL. Exame parasitológico das fezes. "Série Clínica Médica", 4ª edição, Sarvier, São Paulo, 1980.

2. Araújo CFFM. Aspectos teórico-práticos do emprego da técnica de Kato \& Miura em coproscopia quantitativa. Dissertação de Mestrado, Faculdade de Ciências Farmacêuticas da Universidade de São Paulo, São Paulo, SP, 1982.

3. Barreto ML, Smith DH, Sleigh AC. Implications of faecal egg count variation when using the Kato-Katz method to assess Schistosoma mansoni infections. Transactions of the Royal Society of Tropical Medicine and Hygiene 84: 545-555, 1990.

4. Chaia G, Chaia ABMC, Mc Aullife J, Katz N, Gasper D. Coprological diagnosis of schistosomiasis. II. Comparative studies of quantitative methods. Revista do Instituto de Medicina Tropical de São Paulo 10: 349-353, 1968.

5. Chieffi PP, Kanamura H. Diagnóstico laboratorial da esquistossomose mansônica. Revista Brasileira de Malariologia e Doenças Tropicais 30: 77-97, 1978
6. Centro de Informações da Saúde. Manual de Vigilância Epidemiológica. Normas e Instruções. Secretaria de Estado da Saúde, São Paulo, 1978.

7. Coura JR, Conceição MJ. Estudo comparativo dos métodos de Lutz, Kato e Simões Barbosa no diagnóstico coprológico da esquistossomose mansoni. Revista da Sociedade Brasileira de Medicina Tropical 8: 153-158, 1974.

8. Dantas AMP, Ferreira LF. Sôbre o método de Kato no diagnóstico da esquistossomose mansoni. Revista da Sociedade Brasileira de Medicina Tropical 7: 209-212, 1971.

9. Engels D, Sinzinkayo E, Gryssels B. Day-to-day count fluctuation in Schistosoma mansoni and its operational implications. American Journal of Tropical Medicine and Hygiene 54: 319-324, 1996.

10. Gryssels B. Uncertainties in the epidemiology and control of schistosomiasis. American Journal of Tropical Medicine and Hygiene 55: 103-108, 1996.

11. Hoffman VA, Pons JS, Janer JL. Sedimentation concentration method in the schistosomiasis mansoni. Puerto Rico Journal of Public Health Tropical 9: 283-298, 1934. 
12. Kato K, Miura M. Comparative examinations. Japanese Journal of Parasitology 3: 35, 1954.

13. Katz N, Chaves A, Pellegrino J. Simple device for quantitative stool thick-smear technique in schistosomiasis mansoni. Revista do Instituto de Medicina Tropical de São Paulo 14: 397-400, 1972.

14. Komiya Y, Kobayashi A. Evaluation of Kato's thick smear technique with a cellophane cover for helminth eggs in feces. Japanese Journal Medical Science Biological 19: 59-64, 1966.

15. Lutz A. O Schistosomum mansonie a schistosomatose, segundo observações feitas no Brazil. Memórias do Instituto Oswaldo Cruz 11: 121-155, 1919.

16. Martin LK, Beaver PC. Evaluation of Kato tick-smear technique for quantitative diagnosis of helminth infections. American Journal of Medical and Tropical Hygiene 17: 382-391, 1968.

17. Molina J. A técnica de Kato para o diagnóstico coprológico das infecções parasitárias. Revista da Sociedade Brasileira de Medicina Tropical 5: 17-20, 1971.
18. Mott KE. Schistosomiasis control. In: The Biology of Schistosomes. Academic Press, New York, p. 431-450, 1987.

19. Rabelo ALT. Revisão crítica dos métodos de diagnóstico da esquistossomose mansoni. Revista da Sociedade Brasileira de Medicina Tropical 27(supl IV): 177-180, 1994.

20. Superintendência de Campanhas de Saúde Pública. SUCAM. Esquistossomose mansônica. Brasil, Ministério da Saúde, Brasília, DF, 1988.

21. Superintendência de Controle de Endemias. SUCEN. Situação da Esquistossomose no Estado de São Paulo. Secretaria de Estado da Saúde, São Paulo, SP, 1982.

22. World Health Organization. The Control of Schistosomiasis. Technical Report Series N 728. WHO, Geneva, 1985.

23. World Health Organization. Ultrasound in schistosomiasis. TDR/ STR/SCH/00.1. WHO, Geneva, 2000. 